\title{
The Role of Parietal Cortex in Verbal Working Memory
}

\author{
John Jonides, ${ }^{1}$ Eric H. Schumacher, ${ }^{1}$ Edward E. Smith, ${ }^{1}$ Robert A. Koeppe, ${ }^{2}$ Edward Awh, ${ }^{3}$ \\ Patricia A. Reuter-Lorenz, ${ }^{1}$ Christy Marshuetz, ${ }^{1}$ and Christopher R. Willis ${ }^{1}$ \\ ${ }^{1}$ Department of Psychology and 2Division of Nuclear Medicine, Department of Internal Medicine, University of Michigan, \\ Ann Arbor, Michigan, 48109-1109, and 32Department of Psychology, University of California, San Diego, California
}

Neuroimaging studies of normal subjects and studies of patients with focal lesions implicate regions of parietal cortex in verbal working memory (VWM), yet the precise role of parietal cortex in VWM remains unclear. Some evidence (Paulesu et al., 1993; Awh et al., 1996) suggests that the parietal cortex mediates the storage of verbal information, but these studies and most previous ones included encoding and retrieval processes as well as storage and rehearsal of verbal information. A recent positron emission tomography (PET) study by Fiez et al. (1996) isolated storage and rehearsal from other VWM processes and did not find reliable activation in parietal cortex. This result suggests that parietal cortex may not be involved in VWM storage, contrary to previous proposals. However, we report two behavioral studies indicating that some of the verbal material used by Fiez et al. (1996) may not have required phonological representations in VWM. In addition, we report a PET study that isolated VWM encoding, retrieval, and storage and rehearsal processes in different PET scans and used material likely to require phonological codes in VWM. After subtraction of appropriate controls, the encoding condition revealed no reliable activations; the retrieval condition revealed reliable activations in dorsolateral prefrontal, anterior cingulate, posterior parietal, and extrastriate cortices, and the storage condition revealed reliable activations in dorsolateral prefrontal, inferior frontal, premotor, and posterior parietal cortices, as well as cerebellum. These results suggest that parietal regions are part of a network of brain areas that mediate the short-term storage and retrieval of phonologically coded verbal material.

Key words: neuroimaging; parietal cortex; positron emission tomography; retrieval processes; speech; verbal working memory
Working memory involves the storage and manipulation of limited information for a brief time. A particularly human form of working memory that appears to play a significant role in language comprehension and problem-solving is verbal working memory (Jonides, 1995). According to at least one influential model, verbal working memory has three components: a buffer to store phonological codes, a rehearsal process to refresh those codes, and a set of executive processes to manipulate the contents of the buffer in the service of higher cognitive demands (Baddeley, 1992).

Neuroimaging techniques indicate that brain activations accompanying verbal working memory are found in dorsolateral prefrontal, inferior frontal, supplementary motor, premotor, and parietal cortices. Figure 1 summarizes these studies by showing superimposed coordinates of activation from published studies of verbal working memory. One area of activation that is seen repeatedly in these studies is parietal cortex, in the regions labeled by Brodmann as 7 and 40. This area is characteristic of studies of working memory, but not long-term memory, as shown by the analysis of Cabeza and Nyberg (1997). What is the function of this parietal region of activation? Some have argued that it is the seat of storage processes (Paulesu et al., 1993; Awh et al., 1996). However, two experiments that recruited storage processes did not find any evidence of parietal activation at all. How is this to be reconciled?

\footnotetext{
Received Oct. 17, 1997; revised April 8, 1998; accepted April 9, 1998.

This work was supported in part by a grant from the Office of Naval Research and by a grant from the National Institute on Aging, both to the University of Michigan. We thank David Badre, Anna Cianciolo, and Leon Gmeindl for their help in data collection and data analysis.

Correspondence should be addressed to Dr. John Jonides, Department of Psychology, University of Michigan, 525 East University Avenue, Ann Arbor, MI 48109-1109.

Copyright (ㄷ) 1998 Society for Neuroscience $\quad 0270-6474 / 98 / 185026-09 \$ 05.00 / 0$
}

One experiment by Grasby et al. (1993) suffered from a comparison of memory and control conditions tested on different subjects. This may have resulted (as the authors themselves recognized) in insufficient statistical reliability to reveal underlying activations that may have been revealed by within-subject comparisons. The second exception to the finding of parietal involvement in working memory is an experiment that was conducted to isolate the storage processes of verbal working memory (Fiez et al., 1996). Subjects encoded five word or nonword items and were scanned using positron emission tomography (PET) during a 40 sec retention interval only; they recalled the items after scanning was complete. Fiez et al. (1996) reported activations in dorsolateral prefrontal cortex, supplementary motor cortex, and left cerebellum, consistent with other studies represented in Figure 1. Also, they reported activations, although not statistically reliable, in Broca's area and right cerebellum that are also consistent with previous reports. Importantly, however, they reported no reliable activation in parietal cortex even with a region-of-interest analysis designed to focus on this area. This failure to find parietal activation has two possible implications for the nature of processing in verbal working memory tasks: parietal mechanisms are not involved in storage; rather, they are involved in encoding or retrieval processes. We investigated both implications.

First, we sought behavioral evidence that the failure to find parietal activation in the experiment by Fiez et al. (1996) may have been a function of their experimental procedure. Second, we conducted a neuroimaging experiment that replicated and extended the design of the study by Fiez et al. (1996) but also included conditions to assess the contribution of parietal activation to encoding and retrieval processes. 


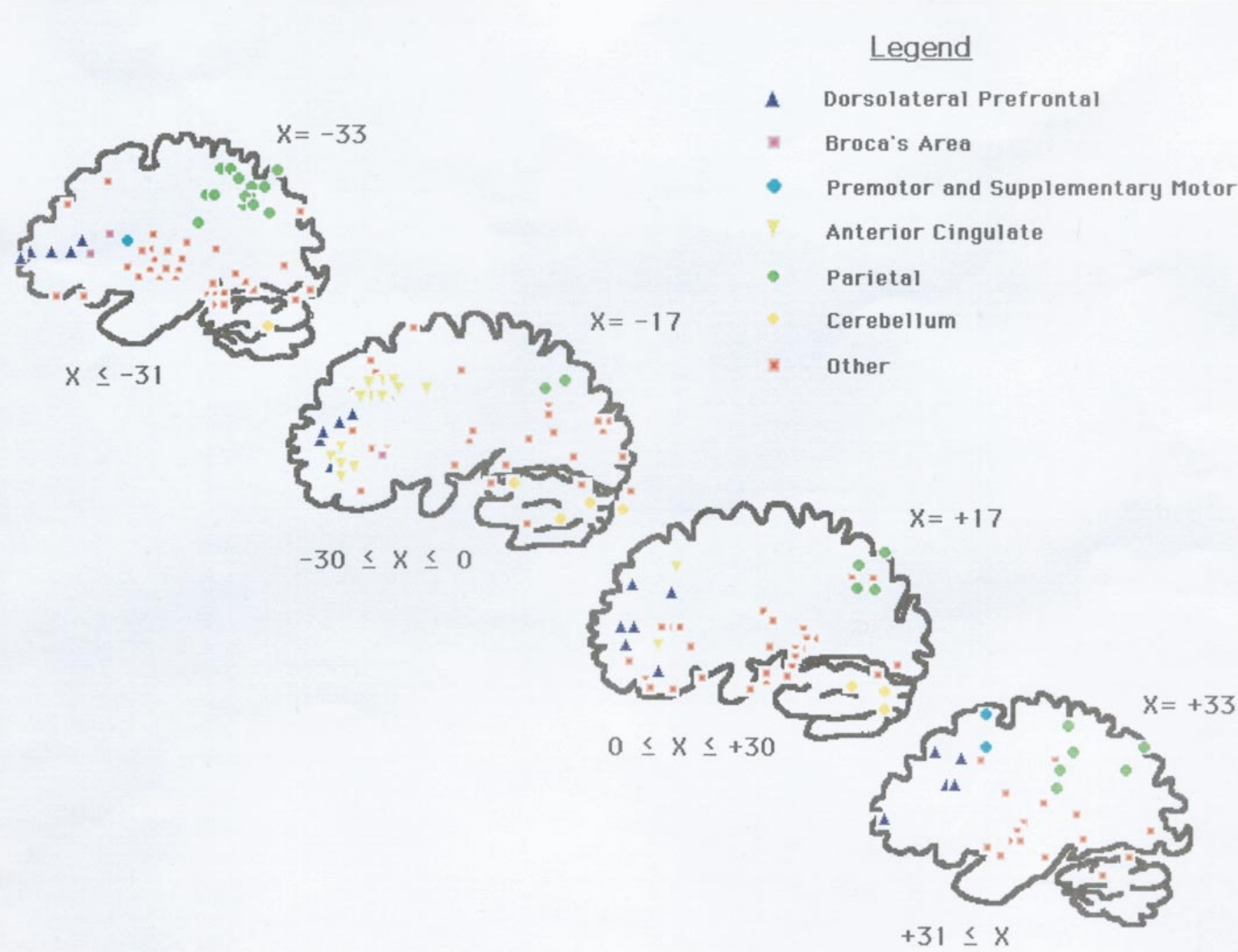

Figure 1. Peak activation pixels reported in neuroimaging studies of verbal working memory (Grasby et al., 1993; Paulesu et al., 1993; Petrides et al., 1993; Andreasen et al., 1995; Awh et al., 1996; Fiez et al., 1996; Schumacher et al., 1996; Smith et al., 1996; Cohen et al., 1997; Jonides et al., 1997). Shown are four parasagittal slices (Tailarach and Tournoux, 1988) in the left $(x=-33$ and $x=-17)$ and right $(x=+17$ and $x=+33)$ hemispheres to provide approximate hemispheric localization of the identified areas. In each slice, reliable areas of activation from the cited reports are localized in proper $y$ and $z$ coordinates, with a range of $x$ coordinates being included in each depicted slice, as indicated at the bottom of each schematic. The different symbols represent different areas of activation, as indicated in the legend. The areas of activation that are taken from each report are not uniquely identified.

\section{BEHAVIORAL EXPERIMENTS}

The experiment by Fiez et al. (1996) included three scans in which retention was required: one for semantically related words, one for unrelated words, and one for nonwords. Words can be coded in terms of some combination of semantic, phonological, or visual codes. However, verbal working memory is typically identified with a phonological coding strategy, a strategy that extends not only to meaningful words but also to other verbal material that is not semantically codable (e.g., syllables). For example, many studies have shown selective interference on material in verbal working memory by phonologically interfering tasks, and others have shown that phonologically similar items are more difficult to store in verbal working memory than dissimilar items (Conrad, 1964; Murray, 1968). Also, patients who have deficits in verbal working memory can be quite normal in their storage of semantic and visual codes (Warrington et al., 1971; Saffran and Marin, 1975). All of this evidence implicates a phonological code in many verbal working memory tasks, a code that may be respon- sible for parietal activations. If, however, subjects in the experiment by Fiez et al. (1996) relied on semantic codes in part to retain the word stimuli, then the impact of a phonological working memory would be mitigated, leaving only one scan (that with nonwords) to show the full activation pattern that might be characteristic of phonological processing. One scan might not be sufficiently sensitive to reveal this pattern. To test the possibility that subjects rely more on phonological working memory for nonwords than words, we first conducted two behavioral experiments, both of which were intended to reveal the use of a phonological coding strategy if one was in play.

\section{EXPERIMENT 1}

\section{Materials and methods}

In the first experiment, we tested subjects on working memory for words and nonwords using a presentation and storage procedure similar to that of Fiez et al. (1996) but with retrieval assessed by a recognition rather than a recall test. A recognition test allowed us to present distractors that 
varied in their similarity to the target items so that we could assess which kinds of distractors were likely to cause errors. From this, we could make inferences about the kinds of internal codes used to store words and nonwords.

Subjects. Twelve right-handed subjects (six males and six females) were paid for participation in an experimental session of $\sim 1 \mathrm{hr}$. All were informed of the experimental procedure and consented to participate.

Stimuli. The experiment consisted of 22 trials, 11 using words as the target stimuli and 11 using nonwords. The words were selected from the list by Paivio et al. (1968), with some additional words that had fewer than seven letters and were of two or three syllables in length; the criterion for selection was words with high frequency of occurrence in English and low concreteness (to mitigate the use of a mnemonic strategy based on imagery). Words in a list were semantically unrelated to one another. The semantically related distractors for the words were chosen from the same categories as the target words. The phonologically similar distractors were chosen so that they either rhymed with one of the targets or were otherwise phonologically similar (e.g., loss-cost). The nonwords were constructed so as not to approximate English words. Phonologically similar nonword distractors were constructed by changing some of the consonants and/or vowels of the target nonwords; they either rhymed with a target or were otherwise phonologically similar, as with the words.

Experimental procedure. Subjects were seated $\sim 46 \mathrm{~cm}$ from a computer monitor. Each trial began with a fixation dot that appeared in the center of the monitor controlled by a Macintosh IIC computer running Psyscope control software (Cohen et al., 1993). The fixation dot remained on the screen throughout the trial. At the beginning of each trial, five targets (either all words or all nonwords) were presented sequentially just below the fixation dot for $3 \mathrm{sec}$ each with $500 \mathrm{msec}$ intervening between successive stimuli, replicating the presentation conditions of Fiez et al., (1996). After presentation of all targets, a tone sounded signaling the beginning of a $55 \mathrm{sec}$ retention interval. The termination of this interval was signaled by a pair of tones after which 22 probe items were presented consecutively just below fixation, each for $500 \mathrm{msec}$ with an interprobe interval of $1500 \mathrm{msec}$. Subjects were to respond with one of two key presses to indicate whether each probe matched one of the five target items. Of these 22 probes, 10 matched the targets (two matches of each target), and 12 were distractor items. When words were the targets, four of the distractors were phonologically similar to one of the targets, four were semantically similar, and four were dissimilar from all targets. When nonwords were the targets, six of the distractors were phonologically similar to one of the targets, and six were dissimilar (of course, there could be no semantically similar distractors for nonwords). The order in which probes of these various types were presented was randomly determined. Half of the subjects received all of the trials with words before any trials with nonwords; the other half were given the trials in the reverse order.

\section{Results and discussion}

When words were the memorized targets, the accuracies of recognition responses to dissimilar distractors, semantically similar distractors, and phonologically similar distractors were 99.2, 98.1, and $99.6 \%$, respectively; the corresponding response times for these three types of distractors were 618, 635, and $665 \mathrm{msec}$, respectively. Even as high and uniform as the accuracies appear, there is an overall effect of distractor type on accuracy as assessed by ANOVA $(p<0.002)$. This overall effect is accounted for by the fact that the semantically similar distractors produced responses less accurate than either the dissimilar $(p<0.001)$ or the phonologically similar distractors $(p<0.001)$. The response times are also different from one another $(p<0.0005)$, and this difference is a result of a reliable difference between the dissimilar distractors and the phonologically similar ones $(p<0.0005)$ as well as a marginally reliable difference between the dissimilar distractors and the semantically similar ones $(p=0.07)$.

When nonwords were the targets, there was a large difference in both accuracy and response time between dissimilar distractors and phonologically similar distractors (96.9 vs $83.8 \%$ and 670 vs $798 \mathrm{msec}$, respectively). The differences between these types of distractors in both accuracy $(p<0.0001)$ and response time $(p<$ $0.0001)$ were highly reliable.
Overall, the word targets showed evidence of both semantic and phonological coding, in that there were effects of semantically similar distractors on accuracy, and of both semantically similar and phonologically similar distractors on response time. For the nonwords, there was a large effect of phonological similarity on both accuracy and response time, an effect that was much larger than the effect of phonologically similar distractors on accuracy or response time for words. This was supported by two ANOVAs (one for accuracy and one for response time) that included words versus nonwords as stimuli and phonologically similar versus dissimilar distractors; these analyses showed that the effect of phonological similarity was decidedly larger for nonwords than for words ( $p<0.0005$ for each of the dependent measures). These data suggest three things about the use of words and nonwords in a paradigm such as that used by Fiez et al. (1996): (1) words are coded and stored using some combination of phonological and semantic representations; (2) nonwords are coded and stored using a phonological representation; and (3) nonwords rely much more on a phonological representation than words. There are two interpretations of the greater effect of phonological similarity on nonwords than words: there is more phonological coding for nonwords than there is for words, and nonwords are not subject to other types of coding that are available for words (e.g., semantic), so the phonological coding effect is more prominent for nonwords. Whichever of these is correct, our results led us to hypothesize that Fiez et al. (1996) may not have found evidence of posterior parietal activation, because their imaging data were heavily influenced by the processes responsible for storing words, and these may have relied more on a semantic than a phonological code. If posterior parietal mechanisms are specific to phonological coding and storage, then this would mitigate activations in this area for word stimuli in their study. To provide converging evidence about this hypothesis, we conducted a second behavioral experiment. This experiment assessed the use of a phonological code by attempting to interfere with its operation.

\section{EXPERIMENT 2}

\section{Materials and methods}

The second experiment was also intended to probe for the use of a phonological working memory strategy when words versus nonwords were used as stimuli. In all respects, the present experiment replicated the presentation and recall parameters of Fiez et al. (1996), with two exceptions. During the retention interval ( $55 \mathrm{vs} 40 \mathrm{sec}$ ), used by Fiez et al. (1996) for technical reasons), we had subjects either engage in articulatory suppression by repeatedly saying "one, two, three, four" aloud at a rate of $\sim 1.5$ digits $/ \mathrm{sec}$, or we had them trace a figure eight repeatedly with their left index finger around a block outline figure eight. Articulatory suppression is known to suppress the ability of subjects to engage in verbal rehearsal, and thus it should interfere with storage and rehearsal of a phonological code if one is in use (Murray, 1968). Thus, we examined whether storage of words versus nonwords was impaired by articulatory suppression, and we compared this effect with any generally interfering effect caused by a secondary task (tracing) that should result in only nonspecific interference with verbal memory.

Subjects. Six subjects participated for payment; all consented to volunteer for an experimental session of $1 \mathrm{hr}$.

Stimuli. The words and nonwords that were used as target stimuli were selected and constructed as described for experiment 1 . In experiment 2 , however, the words that were targets could either be related to one another or unrelated. When related, the words were chosen from the same semantic category; when unrelated, they had no obvious semantic relationship to one another.

Experimental procedure. Subjects were tested on their memory for nonwords, related words, and unrelated words. The order in which these three types of stimuli were tested was varied across subjects in a coun- 
terbalanced manner. For each type of material, subjects were sequentially presented five items of the appropriate type ( $3 \mathrm{sec}$ each with 500 msec intervening between successive items), and they retained those items in memory during a $55 \mathrm{sec}$ retention interval that was filled with one of the two types of interfering task. After this interval, subjects were required to free recall the items orally (as in Fiez et al., 1996), and their responses were recorded for later transcription.

Subjects completed all types of trials with one interfering task and one type of stimulus material (e.g., articulatory suppression interfering with nonword stimuli) before moving on to the other type of interfering task with the same type of stimulus material (e.g., tracing interfering with nonword stimuli). For each interfering task, subjects were given one trial of practice before being given four trials of testing with that interfering task, after which they switched to the other interfering task with the same type of stimulus material. The order in which they were given the two types of interfering tasks was the same for each of the types of stimulus materials they memorized, and the order of interfering tasks was counterbalanced across subjects.

\section{Results and discussion}

The mean recall accuracies for related words, unrelated words, and nonwords were 98, 83, and 51\%, respectively. Overall accuracy was $84 \%$ with the interfering tracing task but only $69 \%$ with the articulatory suppression task. An ANOVA that included type of material (related words, unrelated words, and nonwords) and type of interfering task (tracing and suppression) indicated that both of these main effects were reliable $(p<0.0005$ and 0.02 , respectively). The interaction between these factors was also significant $(p<0.02)$, so we examined the individual effects to locate the source of the reliable interaction. This analysis revealed that articulatory suppression interfered more than tracing for both nonwords and unrelated words (41 vs $61 \%$ for nonwords, $p<0.01 ; 71$ vs $94 \%$ for unrelated words, $p<0.0005)$.

It is surprising that this interference is not greater for nonwords in view of the results of experiment 1 that nonwords showed a greater phonological similarity effect than words. However, in that the performance levels with words and nonwords are quite different overall, it is difficult to conclude much about the apparently equivalent magnitudes of the effects of articulatory suppression. The issue is one of scaling. At a high accuracy level, a $23 \%$ difference may not be psychologically equivalent to a $20 \%$ difference at a lower accuracy level. Consider, for example, rescaling the results into a percentage score for the effect of articulatory suppression. For unrelated words, articulatory suppression produces a decrement in performance of $24 \%(94-71 / 94 \%)$, whereas for nonwords, it produces a decrement of $33 \%$. Looked at in this way, nonwords do show a greater effect with articulatory suppression. Overall, without a theory of what the proper scale of comparison is, it is difficult to evaluate this issue.

For the related words the type of interfering task had no reliable effect ( $97 \mathrm{vs} 98 \% ; p=0.73$ ). We conclude from this that a phonologically interfering task has a pronounced effect on memory for nonwords and for unrelated words, suggesting that a phonological code is used at least in part to retain these stimuli in memory. For related words, the fact that performance is so good overall and that it is not differentially affected by phonological versus nonphonological interfering tasks is consistent with the conclusion that the storage of these materials in memory may rely largely, or perhaps exclusively, on a semantic, rather than a phonological, code.

Taken together, experiments 1 and 2 make the case that the storage of nonwords relies unequivocally on the use of a phonological code. For words, by contrast, storage may rely on both a phonological code and a semantic code. Thus, the use of words in an experiment that investigates phonological working memory may not provide the best evidence of the circuitry that accompanies phonological coding and storage, although it may be entirely suitable to provide evidence of circuitry involved in semantic working memory. For two of its three scans, the experiment by Fiez et al. (1996) relied on words as stimuli; therefore, that experiment may not have had an adequate opportunity to observe brain activations that depended on phonological coding and rehearsal. It should be noted that in a separate analysis, Fiez et al. (1996) did find evidence of greater activation of a frontal opercular region in their nonword than in their word conditions, suggesting that even in their experiment, subjects may have engaged in rehearsal more with nonword than with word stimuli. For this reason, we replicated and extended the experiment by Fiez et al. (1996) using nonwords as stimuli.

\section{NEUROIMAGING EXPERIMENT Materials and methods \\ Rationale}

The neuroimaging experiment was designed to replicate the conditions of Fiez et al. (1996) with sufficient statistical power to discover parietal activation in a storage condition and to add conditions that would assess the role of parietal activation in encoding and retrieval processes as well. We restricted our stimuli to the class of nonwords in this experiment for three reasons: (1) the behavioral data reported above make the case that nonwords evoke a phonological coding and storage strategy, whereas words are subject to semantic coding (in addition to phonological coding); (2) the introspections of subjects in the behavioral experiments indicated that nonwords required much more intensive rehearsal than did words, again suggesting the use of a phonological code for nonwords; and (3) the poorer performance in retrieval of nonwords than words indicates that processing of the former items is more demanding, suggesting that storage and rehearsal might be more effortful with nonwords, thereby leading to greater brain activation in the areas mediating the storage and rehearsal processes. Thus, we were encouraged in the belief that nonwords provided the best opportunity to discover evidence of parietal involvement in verbal working memory using a phonological code.

\section{Subjects}

The participants were six males and six females, all of whom were right-handed. They were paid for their participation, and they consented to participate for two sessions, one that provided practice on the tasks and one during which the PET scans were conducted.

\section{Conditions}

There were three memory and two control conditions in the experiment, each tested twice during the PET session for a total of 10 scans. Each condition was also tested during a practice session that preceded the day of PET testing. The three memory conditions all included nonwords as stimuli, with scanning directed at storage, encoding, or retrieval phases of processing, respectively. The two control conditions were designed to remove effects of perceptual and motor processes.

Storage condition. This condition was designed to be similar to that of Fiez et al. (1996). A trial began with the presentation of five nonwords for $3 \mathrm{sec}$ each, with $500 \mathrm{msec}$ intervening between successive nonwords, for a total presentation interval of $17.5 \mathrm{sec}$. Immediately after this interval, a tone sounded and a fixation cross appeared on the computer screen, which signaled a retention interval that lasted 65 sec. After this, a retrieval phase began with the presentation of a pair of tones, which indicated to subjects that they were to recall the five nonwords in any order they chose. PET scanning began $5 \mathrm{sec}$ after the onset of the retention interval and terminated before the beginning of retrieval. Thus, there was no encoding of stimuli during the period of scanning; the scanning interval was occupied largely by processes of storage and possibly some processes of covert retrieval before the explicit retrieval task.

Encoding condition. In the encoding condition, seven nonwords were presented for $2 \mathrm{sec}$ each with $500 \mathrm{msec}$ intervening between presentation of successive stimuli (seven items were used rather than five, as in the storage condition, to occupy encoding processes more fully in this task). Each nonword was presented twice for a total of $35 \mathrm{sec}$ devoted to presentation of stimuli. After this interval, a tone sounded and a fixation cross appeared on the screen for $65 \mathrm{sec}$, during which time subjects were 
to store the nonwords in memory (just as they did in the storage condition). After this retention interval, a pair of tones sounded, and subjects engaged in retrieval, which consisted of a recognition task (recognition was used to collect data about whether the phonological similarity of mismatches affected responses, as it should if subjects were engaged in phonological encoding). During the retrieval interval, 21 nonwords appeared one at a time, and subjects responded with one of two key presses to indicate whether each presented nonword matched one of the studied items. Seven of these stimuli matched the original nonwords; seven mismatches were phonologically dissimilar; and seven mismatches were phonologically similar to the original nonwords. Each of these test stimuli was presented for $2 \mathrm{sec}$, with $500 \mathrm{msec}$ intervening between successive stimuli. Thus, the retrieval phase as a whole lasted $52.5 \mathrm{sec}$. Scanning was initiated $5 \mathrm{sec}$ before the beginning of the study phase of the experiment and during the first $25 \mathrm{sec}$ of the retention interval when the fixation cross was on the screen (i.e., the scans included encoding processes of interest and then switched to a neutral condition after $35 \mathrm{sec}$; this paradigm has been shown to be effective in revealing activating effects of the task of interest) (Koeppe et al., 1994; Cherry et al., 1995). Thus, the PET data include activations that were largely a function of processes used to encode the stimuli into memory and to store them, with little or no contribution of retrieval processes.

Retrieval condition. We began each trial with the presentation of five nonwords for $3 \mathrm{sec}$ each with $500 \mathrm{msec}$ between successive nonwords (as in the storage condition). This presentation phase was followed by a single tone that signaled a retention interval of $65 \mathrm{sec}$, during a which a fixation cross was presented on the screen (as in the encoding and storage conditions). After the retention interval, a pair of tones was sounded signaling the beginning of retrieval. During the retrieval phase, 14 nonwords were presented for $2 \mathrm{sec}$ each with $500 \mathrm{msec}$ between successive nonwords for a total of $35 \mathrm{sec}$. Of these 14 nonwords, five were identical to the nonwords that subjects stored in memory, five were phonologically similar distractors, and four were phonologically dissimilar distractors. After the last of these nonwords was presented, a fixation dot appeared on the screen and remained for $30 \mathrm{sec}$. Scanning was initiated $5 \mathrm{sec}$ before the beginning of the retrieval phase and continued through the duration when the dot appeared on the screen, for a total of $65 \mathrm{sec}$ (again, making use of a switching paradigm during scanning) (Koeppe et al., 1994; Cherry et al., 1995). In this way, we designed this condition to concentrate on activations that were a function of retrieval processes, but, of course, encoding and storage processes were also involved, in that subjects had to encode the probes and had to continue storing the five target items until the end of the retrieval period.

Fixation control. This condition was designed to match the perceptual and response characteristics of the storage condition and to be similar to the control condition of Fiez et al. (1996). Accordingly, it included just a fixation cross that remained on the screen with no response requirement on the part of subjects. The fixation cross was presented before the beginning of the scan and remained for a total of $105 \mathrm{sec}$, lasting well past the end of the scan.

Nonword control. This condition was designed as a control for the encoding and the retrieval conditions. The condition began with 14 nonwords, each presented for $2 \mathrm{sec}$ with $500 \mathrm{msec}$ between successive nonwords. Subjects were required to push a response button at the onset of each nonword. After the last nonword was presented, a fixation cross appeared on the screen and remained for the duration of the scanning interval. Thus, this condition included presentation of exactly the same number of nonwords as the encoding and retrieval conditions, and it also included a switch to a fixation cross after the 14 nonwords appeared, matching the presentation parameters of the encoding and retrieval conditions. We had subjects make a manual response with the presentation of each nonword to match the responses that they made in the retrieval condition (although this made the control not completely comparable to the encoding condition in which no manual responses were made during the scanning interval).

Conditions were counterbalanced for presentation in the experiment according to the following scheme: each condition was tested once before any condition was tested a second time. The order of presentation of conditions during the first five scans was repeated for the next five. For the first five scans, either the fixation control or the nonword control was presented first, with the other control condition presented fifth in order. Half of the subjects had the fixation control presented first, and half had the nonword control. The middle three scans of the first five were the encoding, storage, and retrieval conditions, with the order among these three counterbalanced completely within each set of three subjects.
Below, we describe the events for a scan of each of the three memory conditions and the two controls.

\section{PET protocol}

A Siemens (Erlangen, Germany) ECAT EXACT-47 PET scanner was used. It produced 47 contiguous slices that were $3.375 \mathrm{~mm}$ (center to center) apart. The reconstructed in-plane resolution was $10 \mathrm{~mm}$ full-width, half-maximum. Subjects were positioned in the scanner, and their head positions were recorded. Head position was verified before each scan. Each subject had an intravenous catheter inserted into his or her right arm.

The experimenter administered an intravenous bolus of 40 $\mathrm{mCi}$ of $\left[{ }^{15} \mathrm{O}\right]$ water at the beginning of each scan as the subject began the sequence of trials. PET scanning began $5 \mathrm{sec}$ after the count rate increased above the background level and continued for $60 \mathrm{sec}$. Twelve minutes separated each scan to allow the background radiation to reach an $\sim 1 \%$ level caused by clearance and decay.

\section{Results and discussion}

\section{Behavioral data}

Accuracy for the storage condition, in which free recall was the required retrieval task, was $64 \%$ during the PET session, somewhat lower than that of Fiez et al. (1996), in which a response had to match one of the encoded items perfectly to be scored correct. For the encoding and retrieval conditions, for which the retrieval task was recognition, average accuracies were 93 and $92 \%$, respectively, during the PET session and 88 and $94 \%$ during the practice session. For these tasks also, we recorded response times, which averaged 889 and $880 \mathrm{msec}$ for the encoding and retrieval conditions, respectively, during the PET session and 886 and 870 msec during the practice session.

Of interest is a comparison of responses when a probe item on the recognition test in the encoding and retrieval conditions did not match one of the target nonwords. A mismatch could be either phonologically similar to a target or dissimilar from all targets in the set for that trial. When a probe was similar to the target, accuracy and response times were reliably worse than when the probe and target were not similar in both the PET and practice sessions. Participants were $13 \%$ less accurate when the probe was similar to the target than when it was not similar during the PET session (99\% for dissimilar and $86 \%$ for similar probes; $p<0.0001)$ and $15 \%$ less accurate during the practice session (99\% for dissimilar and $84 \%$ for similar probes; $p<0.002$ ) (the data from one participant during the practice scan were not analyzed because of a technical error). Participants were 172 msec slower when the probe was similar to the target than when it was not during the PET session (805 msec for dissimilar and 977 msec for similar probes; $p<0.0001)$ and 138 msec slower during the practice session $(809 \mathrm{msec}$ for dissimilar and $947 \mathrm{msec}$ for similar probes; $p<0.0001)$. These effects, for both response time and accuracy, were not reliably different for the encoding and retrieval conditions. Thus, there is a pattern of phonological similarity effects in these data that mimics that in experiment 1 , suggesting, once again, that subjects encode and store phonological representations for nonwords in this task.

\section{PET data}

We conducted omnibus analyses of each of the memory conditions compared with its appropriate control as well as specific analyses that asked whether there was activation in posterior parietal and other regions. For the omnibus analyses, regional cerebral blood flow changes between two different conditions 
Table 1. Significant activation and deactivation foci in the experimental - control subtractions

Stereotaxic Coordinates

\begin{tabular}{|c|c|c|c|c|}
\hline$x$ & $y$ & $z$ & $Z$ Score & Brain Area \\
\hline \multicolumn{5}{|c|}{ Storage - fixation } \\
\hline 33 & 53 & 7 & 4.72 & Right dorsolateral frontal (area 46) \\
\hline 35 & 39 & 29 & 6.97 & Right dorsolateral frontal (area 46) \\
\hline-35 & 35 & 25 & 4.43 & Left dorsolateral frontal (area 46) \\
\hline 30 & 21 & 4 & 5.07 & Right inferior frontal (area 45) \\
\hline-30 & 12 & 7 & 4.94 & Left broca's area (area 44) \\
\hline-53 & -1 & 34 & 5.70 & Left premotor (area 6) \\
\hline-3 & -4 & 56 & 4.86 & SMA (area 6) \\
\hline-28 & -49 & -25 & 4.93 & Left cerebellum \\
\hline 42 & -51 & 40 & 5.16 & Right posterior parietal (area 40) \\
\hline 21 & -55 & -20 & 6.49 & Right cerebellum \\
\hline-39 & -60 & -18 & 4.70 & Left cerebellum \\
\hline-3 & 53 & -11 & -8.35 & Orbital frontal (area 11) \\
\hline-1 & 53 & 18 & -7.97 & Anterior frontal (area 9) \\
\hline-8 & 41 & 43 & -5.24 & Anterior frontal (area 8) \\
\hline-10 & 35 & 47 & -5.27 & Anterior frontal (area 8) \\
\hline 42 & 5 & -22 & -4.75 & Right midtemporal (area 21/38) \\
\hline-46 & 3 & -22 & -5.82 & Left midtemporal (area 21) \\
\hline 1 & -51 & 20 & -7.83 & Posterior cingulate (area 30) \\
\hline-46 & -69 & 22 & -5.83 & Left posterior parietal (area 39) \\
\hline \multicolumn{5}{|c|}{ Encoding - nonword } \\
\hline-24 & -91 & -2 & $4.34^{a}$ & Left occipital (area 18) \\
\hline 57 & -33 & 22 & $-3.91^{a}$ & Right posterior parietal (area 40) \\
\hline \multicolumn{5}{|c|}{ Retrieval - nonword } \\
\hline 37 & 53 & -4 & 4.59 & Right anterior frontal (area 10) \\
\hline-51 & 28 & 29 & 4.56 & Left anterior frontal (area 9) \\
\hline 42 & 21 & 22 & 5.34 & Right dorsolateral frontal (area 46) \\
\hline-1 & 10 & 38 & 5.33 & Anterior cingulate (area 32) \\
\hline 39 & -51 & 43 & 5.77 & Right posterior parietal (area 40) \\
\hline 24 & -89 & -9 & 4.36 & Right occipital (area 18) \\
\hline-24 & -91 & -7 & 4.65 & Left occipital (area 18) \\
\hline-44 & -71 & 18 & -5.38 & Left posterior parietal (area 39) \\
\hline 55 & 3 & -16 & -5.12 & Right midtemporal (area 21) \\
\hline-53 & -8 & -18 & -4.53 & Left midtemporal (area 21) \\
\hline 17 & -53 & 18 & -4.58 & Right posterior cingulate (area 23) \\
\hline 51 & -62 & 11 & -6.49 & Right posterior parietal (area 39) \\
\hline
\end{tabular}

Brodmann areas are shown in parentheses. Deactivations are shown in italics.

${ }^{a}$ Sites with highest activation and deactivation levels (not significant).

were assessed by subtraction analysis of PET image sets (Friston et al., 1991; Worsley et al., 1992). First, intrasubject registration by an automated algorithm corrected motion between scans within a subject's PET session (Minoshima et al., 1993a). PET image sets for each subject were then transformed to a standard bicommissural stereotactic system (Talairach and Tournoux, 1988). The transformation procedure consisted of three steps: (1) detection of the midsagittal plane of the brain (Minoshima et al., 1992), (2) detection of the bicommissural (anterior commissural-posterior commissural) line of the midsagittal plane (Minoshima et al., 1993b), and (3) linear scaling followed by nonlinear warping of the brain to remove anatomic differences among subjects (Minoshima et al., 1994). Next, a subtraction image set was created for each subject between the averaged images for memory task minus its control condition. The subtraction image sets were then averaged across subjects, producing a group average subtraction image set consisting of mean and SD of cerebral blood flow $(\mathrm{CBF})$ changes for each brain voxel. SDs for the voxels were averaged within the brain to create a pooled estimate of variance, and $t$ statistical values were calculated for each voxel using a pooled variance estimate and correcting for multiple nonindependent comparisons (Friston et al., 1991; Worsley et al., 1992). Significant CBF changes were localized using stereotactic coordinates and are displayed in the figures on a standard nonlinearly warped magnetic resonance image (to Talairach coordinate space) for visual interpretation.

The areas of reliable activations and deactivations are reported in Table 1 for each of the memory conditions minus its respective control (i.e., when the control condition produced lower activation than the experimental condition or when it produced higher activation than the experimental condition). For each reliable area, we report the stereotactic coordinate, the $Z$ score of the peak pixel, and the Brodmann label for the area.

Storage-fixation control. Consider first the activations and de- 
activations for the storage-fixation subtraction reported in Table 1. This condition closely approximates the condition used by Fiez et al. (1996). We find activation in left inferior frontal gyrus (Broca's area), left premotor cortex, supplementary motor cortex, and right cerebellum, all of which are characteristic of a circuit for verbal working memory (Fig. 1). We also find activation in dorsolateral prefrontal cortex in both left and right hemispheres, a pattern that has also often been associated with some verbal working memory tasks (Smith et al., 1996; Cohen et al., 1997). In addition, there is reliable activation in left cerebellum and in right inferior frontal gyrus, both of which are approximately homologous to activations found in the opposite hemisphere (homologous bilateral activation is often found in studies using working memory tasks; Smith et al., 1996).

In addition to these sites, note also that there is reliable activation in lateral posterior parietal cortex in Brodmann's area 40, an area not found by Fiez et al. (1996). This site of activation is reliably activated by post hoc analysis in the right hemisphere only, but inspection of the activation images suggests that there is also activation of a homologous site in the left hemisphere. Because posterior parietal sites of activation are often found in studies of verbal working memory, we constructed regions of interest (ROIs) based on data from previous verbal working memory experiments to interrogate the present data to determine whether there was statistically reliable activation in both left and right parietal sites. For all of these ROI analyses, we took peak voxels of activation from previous experiments and constructed spherical ROIs with a diameter of $10.8 \mathrm{~mm}$ and placed these on the data of individual subjects from the present experiment. We then averaged the activation of all voxels within the ROI for each subject and compared this mean to zero to determine whether there was significant activation present.

The first such analysis was based on a verbal working memory task from Awh et al. (1996) in which subjects had to match each of a series of letters to the letter that had occurred two items back in the series (a "two-back" task). In this experiment, there were two left and two right parietal sites of activation with coordinates $(-33,-46,38),(-17,-60,45),(12,-64,47)$, and $(26,-55$, and 50). Applying these ROIs to the data of the storage-fixation subtraction revealed two reliable activations $(p<0.05)$, one in each hemisphere $(-33,-46,38 ; 12,-64,47)$. We next created ROIs from a set of experiments that had used a three-back verbal working memory task (matching each letter against the one that was three items back in the series) (Smith et al., 1996; Schumacher et al., 1996, Jonides et al., 1997). Collapsing across these three experiments, two reliable parietal ROIs were chosen, one in the left $(-35,-49,38)$ and the other in the right $(37,-49,40)$ hemisphere. Applying these ROIs to the present data revealed reliable activation in both areas $(p=0.053$ in the left and $p<$ 0.002 in the right hemispheres). Taken together, these analyses reveal that there are sites of reliable activation in posterior parietal cortex, in both the left and right hemispheres. These sites are located either in Brodmann's area 7 or 40 , sites that have previously been implicated in working memory tasks.

We compared the activations in the left and right posterior sites to determine whether one was reliably larger than the other by using the ROIs from the three-back tasks, cited above. Although the magnitude of the activation was larger on the right than on the left, these activations did not differ reliably $(p=0.2351)$. Nonetheless, we are cautious in accepting a null hypothesis about this difference. In part this is because we entertain the hypothesis that some of the parietal activation we obtained may be a function of attentional processes that are required to change focus from one item to another as they are rehearsed. This might be expected to be a function of processes more of right than left parietal cortex, based on imaging studies of selective attention (Corbetta et al., 1993, Heinze et al., 1994). So, the greater magnitude of right than left parietal activation that we have documented, although not reliable, may indicate an involvement of parietal mechanisms in shifting attention from internal representations of one item to another as they are rehearsed. At this point, this issue is not resolved. We do note, however, that our previous studies of verbal working memory have also found substantial right as well as left parietal activation, so our data are not inconsistent with these past results (Awh et al., 1996; Schumacher et al., 1996; Smith et al., 1996; Jonides et al., 1997).

Another focus of interest in the storage-fixation subtraction is the activation in dorsolateral prefrontal areas. Table 1 shows that there was one site of reliable activation in left dorsolateral prefrontal cortex and three sites in right. These regions may be involved in the executive control of processes required for storage and rehearsal of the nonwords in our study. For example, there may be processes necessary to tag the memoranda with relevant context so that they can be retrieved later. Another possibility is that the long retention interval used in our study, and that of Fiez et al. (1996) as well, resulted in the recruitment of long-term memory processes in addition to processes of working memory. If this were the case, we might expect that there would be some overlap between the dorsolateral prefrontal areas that we observed and those frequently found in other long-term memory studies. Of course, areas involved in the encoding of information for long-term memory are not relevant to this issue in that the storage condition of the present experiment did not involve any encoding at all. However, regions of activation in long-term memory experiments that may be involved in either storage or retrieval may be relevant (assuming that the storage condition of the present experiment may have involved some covert, internal retrieval as well). With this in mind, we used the 10 sites of activation that Cabeza and Nyberg (1997) identified as sites from long-term memory studies that may have been involved in storage or retrieval (coordinates: $32,7,55 ; 30,32,33 ; 27,47,1 ; 48,8,32$; $26,27,8 ; 32,42,15 ; 28,21,-2 ;-29,53,1 ;-23,25,3$; and -36 , $50,10)$. We then created spherical regions of interest from the peak voxels of these sites with a diameter of $10.8 \mathrm{~mm}$ and applied these to the present data. Eight of the 10 sites had reliable activations $(p<0.05)$, and the other two approached reliability $(p<0.11)$.

Overall, then, the pattern of activation we find in the subtraction of the fixation control from the storage condition is consistent with past studies of verbal working memory, including, importantly, activation in posterior parietal cortex. In addition, we find some evidence that there may be some long-term memory involvement in the storage condition, which we attribute to the long retention intervals involved in the experiment. We conclude that there continues to be reason to suspect involvement of the posterior parietal cortex in storage processes. The involvement may be as part of a circuit controlling shifts of attention involved in rehearsing the verbal material, or it may be as part of a circuit involving storage itself.

Retrieval-nonword control. The activations found in a subtraction of the nonword control from the retrieval condition are also listed in Table 1 . Note that by post hoc analysis there is reliable activation in right posterior parietal cortex in a region almost identical to that found in the storage-fixation control subtraction. 
In addition, there is activation in right dorsolateral prefrontal cortex, overlapping the activation site found in the storagefixation subtraction. This is confirmed by placing ROIs from the parietal and frontal areas of the storage-fixation subtraction on the data of the retrieval-nonword subtraction. In that these two subtractions represent completely independent scans, the fact that there is overlap in the parietal and frontal activations suggests that the storage and retrieval conditions share some circuitry in common. A strong candidate for the function that is mediated by this common circuitry is storage: this is the defining feature of the storage condition, and storage of the target nonwords is required in the retrieval conditions while subjects are matching these targets against the probes that they encounter. It seems reasonable, therefore, to argue that a combination of frontal and parietal mechanisms is involved in storage processes. This argument is consistent with previous research that has also implicated parietal (Paulesu et al., 1993; Smith et al., 1996; Jonides et al., 1997) and frontal (Cohen et al., 1997) mechanisms in the storage of information in working memory.

For the same reason that we suspected that long-term memory processes may have played some role in the storage condition, we also suspected that they may have been involved in the retrieval condition. Consequently, we applied a region-of-interest analysis to the retrieval-nonword activations using the same 10 ROIs identified from the report of Cabeza and Nyberg (1997) that mark long-term memory regions playing a role in storage. Of these 10 regions, eight were reliably activated $(p<0.05)$ in the retrievalnonword subtraction; the remaining two were suggestively close ( $p=0.08$ and 0.14 ). Thus, we suspect that the long retention interval used in the retrieval condition may, as with the storage condition, have permitted long-term memory processes to play some role.

We also note that the retrieval condition shows evidence of activation in left and right extrastriate regions of occipital cortex. Perhaps the control condition did not engage perceptual mechanisms for encoding the nonwords to the same extent as did the retrieval condition. This may have been a result of subjects having to attend to the identity of each nonword that was presented in the retrieval condition but having to attend only to the fact that an item was presented in the control condition, regardless of its identity.

Encoding-nonword control. As Table 1 reports, this subtraction yielded no areas of activation that were statistically significant, but one area of activation in left extrastriate occipital cortex was nearly significant. This site of activation is nearly identical to one of the extrastriate sites found for the retrieval-nonword subtraction, and so we attribute it to the same hypothesized cause, greater encoding processes recruited by the encoding condition than by the control condition.

It is of interest to note that the encoding condition did not show any evidence of posterior parietal activations. This was confirmed by applying ROIs for left and right posterior parietal sites (identified in the same way as in the analysis of the storage-fixation subtraction) to the encoding-nonword subtraction. None of these ROIs revealed significant sites of activation $(p>0.20$ in both cases).

Of course, one might argue that the present paradigm involves not only working memory but also long-term memory processes, as we suggested above. So, perhaps mechanisms that are involved in the encoding of verbal information for long-term storage are also appropriate candidates to reveal themselves in this condition. To examine this, we identified five areas that Cabeza and Nyberg
(1997) identified as associated with long-term encoding, and we applied ROIs based on these areas to the present data. Only one area $(-2,28,28)$ approached reliability $(p=0.056)$.

It is possible that the amount of encoding that was necessary in the encoding condition was relatively light, and so the brain mechanisms that mediated this encoding were not sufficiently taxed to reveal themselves in our study. This possibility is somewhat contradicted by the fact that we did find a site of nearly reliable activation in extrastriate cortex. Nevertheless, we remain cautious in interpreting the null findings of encoding-related activations in either posterior parietal cortex or in areas associated with encoding for long-term memory. Exercising this caution, however, we offer the hypothesis that posterior parietal cortex is not a primary site of mechanisms involved in the encoding of material for working memory.

Deactivations. We have included in Table 1 regions in which the control conditions produced higher relative cerebral blood flow than their respective experimental conditions. There is little general agreement from previous research about the proper interpretation of "deactivations" such as these. As Shulman et al. (1997) discuss, there are two classes of hypothesis to account for them: decreased activation in the memory conditions as a function of inhibitory task processes or suspension of processes in the memory conditions that are normally active in the control conditions. Shulman et al. (1997) point out that task-related deactivations caused by processes of the experimental conditions should be idiosyncratic; that is, they should reflect the specific processes that are needed by these conditions. By contrast, there may be more commonality among the deactivated areas across experiments if these areas represent suspension of processes that are normally active in the passive control conditions (e.g., unconstrained thought processes and monitoring of the external environment). Comparison of the areas of deactivation shown in Table 1 with those documented by Shulman et al. (1997) for nine visual tasks that do not involve working memory explicitly reveals some commonalities and some differences. Broadly speaking, there are similarities in deactivations in areas of medial frontal cortex, posterior cingulate, sections of posterior parietal cortex, and anterior temporal areas. At a finer grain of analysis, though, the match between the deactivations shown in Table 1 and those reported by Shulman et al. (1997) is sporadic. So, it is difficult to conclude whether there is a consistent pattern of deactivations across studies regardless of task or whether the deactivations are task-specific. We do note that many of the regions of deactivation in our experiment 3 are ones that have been cited in previous studies of working memory (Jonides et al., 1997). These include regions of inferior temporal cortex, angular gyrus, precuneus, and frontal pole. Certainly, the issue of whether there is common or distinct deactivation across tasks deserves much further study.

\section{CONCLUSIONS}

Overall, the results of the present study confirm what is by now a growing literature on the circuitry of verbal working memory. This literature has documented the involvement of a network of sites concentrated in dorsolateral prefrontal, premotor, supplementary motor, inferior prefrontal, cerebellar, and posterior parietal areas. The storage condition of the present experiment reveals activations in all of these sites, including the posterior parietal site that was not activated in two previous reports (Grasby et al., 1993, Fiez et al., 1996). We conclude that the pattern of activations we and others have found for parietal cortex implicates it in mechanisms involved in the storage of verbal 
material. Corroborating these observations are several cases in which deficits in verbal working memory can be attributed to lesions in the region of posterior parietal cortex and surrounding tissues, although these lesions are uniformly in left hemisphere, whereas we find activations in both hemispheres (Warrington et al., 1971; Saffran and Marin, 1975; Shallice and Vallar, 1990). The neuroimaging and neuropsychological evidence thus appears to converge on the conclusion that posterior parietal cortex plays a role in the storage of verbal information in working memory. In addition, perhaps, there may also be posterior parietal involvement in attentional mechanisms used in working memory, mechanisms that may be necessary as part of rehearsal processes.

These conclusions expand on the report by Fiez et al. (1996) in an important way. The circuitry for verbal working memory that was documented by these authors, we surmise, was largely a function of the storage of semantically rich words, unlike the nonwords that we used exclusively in our experiment 3 . With such materials, a phonological storage strategy may not play a particularly prominent role; rather, subjects may rely more on semantic codes for the words to store them in working memory. Perhaps then, this implicates posterior parietal mechanisms in the storage of phonological codes in verbal working memory, with nonphonological codes for verbal materials relying on other brain structures. Although there is not a great deal of behavioral evidence to support the use of semantic codes in verbal working memory, there is some evidence (Shulman, 1971) suggesting at least that such codes are possible. Thus, we are left with the conclusion that verbal working memory may be mediated by either phonological or semantic storage and that the role of posterior parietal cortex may hinge on the use of phonological coding.

\section{REFERENCES}

Andreasen NC, O'Leary SO, Arndt S, Cizadlo T, Hurtig R, Rezai K, Watkins GL, Boles Ponto LL, Hichwa RD (1995) Short-term and long-term verbal memory: a positron emission tomography study. Proc Natl Acad Sci USA 92:5111-5115.

Awh E, Jonides J, Smith EE, Schumacher EH, Koeppe RA, Katz S (1996) Dissociation of storage and rehearsal in verbal working memory. Psychol Sci 7:25-31.

Baddeley AD (1992) Working memory. Science 255:556-559.

Cabeza R, Nyberg L (1997) Imaging cognition: an empirical review of PET studies with normal subject. J Cognit Neurosci 9:1-26.

Cherry SR, Woods RP, Doshi NK, Banergee PK, Mazziotta JC (1995) Improved signal-to-noise in PET activation studies using switched paradigms. J Nucl Med 36:307-314.

Cohen JD, MacWhinney B, Flatt MR, Provost J (1993) PsyScope: a new graphic interactive environment for designing psychology experiments. Behav Res Methods Instrum Comput 25:257-271.

Cohen JD, Perlstein WM, Braver TS, Nystrom LE, Noll DC, Jonides J, Smith EE (1997) Temporal dynamics of brain activation during a working memory task. Nature 386:604-608.

Conrad R (1964) Acoustic confusions in immediate memory. Br J Psychol 55:75-84.

Corbetta M, Miezin F, Shulman GL, Petersen SE (1993) A PET study of visuospatial attention. J Neurosci 13:1202-1206.

Fiez JA, Raife EA, Balota DA, Schwarz JP, Raichle ME, Petersen SE (1996) A positron emission tomography study of the short-term maintenance of verbal information. J Neurosci 16:808-822.

Friston KJ, Frith CD, Liddle PF, Frackowiak RSJ (1991) Comparing functional (PET) images: the assessment of significant change. J Cereb Blood Flow Metab 11:690-699.
Grasby PM, Frith CD, Friston KJ, Bench C, Frackowiak RSJ, Dolan RJ (1993) Functional mapping of brain areas implicated in auditory-verbal memory function. Brain 116:1-20.

Heinze HJ, Mangun GR, Burchert W, Hinrichs H, Scholz M, Munte TF, Gos A, Scherg M, Johannes S, Hundeshage H, Gazzaniga MS, Hyllyard SA (1994) Combined temporal imaging of brain activity during visual selective attention in humans. Nature 372:543-546.

Jonides J (1995) Working memory and thinking. In: An invitation to cognitive science: thinking, Vol 3 (Smith EE, Osherson DN, eds). Cambridge, MA: MIT.

Jonides J, Schumacher EH, Smith EE, Lauber E, Awh E, Minoshima S, Koeppe RA (1997) The task-load of verbal working memory affects regional brain activation as measured by PET. J Cognit Neurosci 9:462-475.

Koeppe RA, Minoshima S, Buchtel HA, Botti LE, Henry TR (1994) Enhanced detectability of $\mathrm{CBF}$ changes in PET $\left.]^{15} \mathrm{O}\right]$ water activation studies of human cognition. J Nucl Med 35:138.

Minoshima S, Berger KL, Lee KS, Mintun MA (1992) An automated method for rotational correction and centering of three-dimensional functional brain images. J Nucl Med 33:1579-1585.

Minoshima S, Koeppe RA, Fessler JA, Mintun MA, Berger KL, Taylor SF, Kuhl DE (1993a) Integrated and automated data analysis method for neuronal activation studies using $\left[{ }^{15} \mathrm{O}\right]$ water PET. In: Quantification of brain function: tracer kinetics and image analysis in brain PET (Uemura K, Lassen NA, Jones T, Kanno I, eds), pp 409-418. Tokyo: Excepta Medica.

Minoshima S, Koeppe RA, Mintun MA, Berger KL, Taylor SF, Frey KA, Kuhl DE (1993b) Automated detection of the intercommissural line for stereotactic localization of functional brain images. J Nucl Med 34:322-329.

Minoshima S, Koeppe RA, Frey KA, Kuhl DE (1994) Anatomical standardization: linear scaling and nonlinear warping of functional brain images. J Nucl Med 35:1528-1537.

Murray DJ (1968) Articulation and acoustic confusability in short-term memory. J Exp Psychol 78:679-684.

Paivio A, Yuille JC, Madigan SA (1968) Concreteness, imagery, and meaningfulness values for 925 nouns. J Exp Psychol 76:1-25.

Paulesu E, Frith CD, Frackowiak RSJ (1993) The neural correlates of the verbal component of working memory. Nature 362:342-344.

Petrides M, Alivisatos B, Meyer E, Evans AC (1993) Functional activation of the human frontal cortex during the performance of verbal working memory tasks. Proc Natl Acad Sci USA 90:878-882.

Saffran EM, Marin SM (1975) Immediate memory for word lists and sentences in a patient with deficient auditory short-term memory. Brain Lang 2:420-433.

Schumacher EH, Lauber E, Awh E, Jonides J, Smith EE, Koeppe RA (1996) PET evidence for an amodal verbal working memory system. NeuroImage 3:79-88.

Shallice T, Vallar G (1990) The impairment of auditory-verbal shortterm storage. In: Neuropsychological impairments of short-term memory (Vallar G, Shallice T, eds). Cambridge, UK: Cambridge UP.

Shulman GL, Fiez JA, Corbetta M, Buckner RL, Miezin FM, Raichle ME, Petersen SE (1997) Common blood flow changes across visual tasks: II. Decreases in cerebral cortex. J Cognit Neurosci 9:648-663.

Shulman HG (1971) Similarity effects in short-term memory. Psychol Bull 75:399-415.

Smith EE, Jonides J, Koeppe RA (1996) Dissociating verbal and spatial working memory using PET. Cereb Cortex 6:11-20.

Talairach J, Tournoux P (1988) Co-planar stereotaxic atlas of the human brain. Stuttgart, Germany: Thieme.

Warrington EK, Logue V, Pratt RTC (1971) The anatomical localisation of selective impairment of auditory verbal short-term memory. Neuropsychologia 9:377-387.

Worsley KJ, Evans AC, Marrett S, Neelin P (1992) A three-dimensional statistical analysis for rCBF activation studies in human brain. J Cereb Blood Flow Metab 12:900-918. 